\title{
A SINGULAR PERTURBATION PROBLEM FOR THE LAVRENT'EV-BITSADZE EQUATION*
}

\author{
by R. J. WEINACHT
}

(Received 9th January 1979, revised 3rd June 1981)

\section{Introduction}

In this note we consider a singular perturbation problem for the equation

$$
\left\{\begin{array}{l}
\varepsilon^{2} K(y) u_{x x}+u_{y y}=0, \quad y>0 \\
K(y) u_{x x}+u_{y y}=0, \quad y<0
\end{array}\right.
$$

where $K(y)=\operatorname{sgn} y$ and. $\varepsilon$ is a small (positive) parameter. This equation for $\varepsilon \neq 0$ is elliptic for $y>0$ and hyperbolic for $y<0$. Many of the results carry over to more difficult and interesting problems for equations of mixed type. The particularly simple model treated here permits the elimination of some complications in the analysis involving singular integral equations while preserving the main qualitative features of more general cases. For a special Tricomi-like problem for (1.1) we construct asymptotic expansions in $\varepsilon$, including boundary layer corrections, of the solution. A proof of uniform asymptotic validity of the lowest order terms is given.

For $\varepsilon=1$ equation (1.1) becomes the Lavrent'ev-Bitsadze equation (see [1] and references therein) which has proved to be a reliable simple model for more complicated equations of mixed type, in particular the Chaplygin and Tricomi equations. These equations, of course, are of importance for transonic gas dynamics when studied in the hodograph plane. We do not claim that the perturbation problem treated here has immediate application to that important area.

Despite the intense activity in singular perturbation problems in recent years the author is not aware of any proofs of uniform asymptotic validity in the case of partial differential equations of mixed type.

As in the case of purely elliptic problems (Eckhaus and de Jager [2]) and purely hyperbolic problems (de Jager [3]) the direction of the subcharacteristics (i.e. characteristics of the reduced equation) plays a vital role. References [2]-[4] provide some further references to the vast literature on singular perturbations.

The technique used here is one of multiple scales as introduced by Latta [5] and our approach follows that of Keller [6] in his treatment of a parabolic problem but results can also be obtained by stretching and matching. The new feature here is how the

* Research supported in part by a grant from the Fulbright-Kommission, Germany. 
elliptic and hyperbolic regions interact and, in particular, how the boundary layers in the elliptic region carry over onto the characteristics which bound the hyperbolic region (see the construction in Section 3 and the discussion in Section 5). A shortcoming of our results is that our assumptions on the data may very well suppress corner expansions (as well as corner singularities) which would otherwise be present. It is hoped that attention will be called to these matters and more definitive results will be forthcoming.

\section{Statement of the problem}

By $\Omega$ we denote the region in the $x y$-plane bounded below in the hyperbolic region by the characteristics $C_{0}=\left\{(x,-x): 0 \leqq x \leqq \frac{1}{2}\right\}, C_{1}=\left\{(x, x-1): \frac{1}{2}<x \leqq 1\right\}$ and above in the elliptic region by the straight line segments $\Gamma_{0}=\left\{(0, y): 0 \leqq y<\gamma_{0}\right\}, \Gamma_{1}=\left\{(1, y): 0 \leqq y<\gamma_{1}\right\}$ and the smooth curve $\Gamma=\left\{(x, \gamma(x))\right.$ : $\left.0 \leqq x \leqq 1, \gamma \in C^{\infty}[0,1], \gamma(0)=\gamma_{0}, \gamma(1)=\gamma_{1}\right\}$ which joins the points $\left(0, \gamma_{0}\right),\left(1, \gamma_{1}\right)$ and lies completely above the $x$-axis $(\gamma$ is a strictly positive function). The reason for this choice of region is based upon the fact that it was desired to treat a region closely resembling a Tricomi region but with entire segments of the boundary in the direction of the subcharacteristics. This case was also treated for elliptic problems in [2], [4].

We will denote by $\Omega_{+}$the elliptic region $\Omega_{+}=\{(x, y) \in \Omega: y>0\}$ and by $\Omega_{-}$the hyperbolic region $\Omega_{-}=\{(x, y) \in \Omega: y<0\}$.

Our problem consists of equation (1.1) together with the boundary conditions

$$
\begin{gathered}
\left.u\right|_{c_{0}}=\psi \\
\left.u\right|_{\Gamma}=\phi \\
\left.u\right|_{\Gamma_{0}}=\phi_{0} \\
\left.u\right|_{\Gamma_{1}}=\phi_{1}
\end{gathered}
$$

with the obvious compatibility conditions to ensure continuity of the boundary data.

We seek asymptotic solutions uniformly valid in $\Omega$, i.e. in the maximum norm and hence consider smooth data and seek classical solutions of the boundary value problem (1.1), (2.1)-(2.4) in the class

$$
\mathscr{C}(\Omega):=C^{2}\left(\Omega_{+} \cup \Omega_{-}\right) \cap C^{1}(\Omega) \cap C(\bar{\Omega})
$$

This is precisely the set of classical solutions usually considered when $\varepsilon=1$ ([1]). Existence of a solution $u$ for given data is assumed. Uniqueness follows from Lemma 2 of Section 4.

\section{Formal construction of asymptotic solutions}

Based on results of singular perturbation theory we make the following ansatz for the solution $u$ of our boundary value problem

$$
u(x, y ; \varepsilon) \sim U(x, y ; \varepsilon)+\tilde{V}(x, y ; \varepsilon)+\tilde{W}(x, y ; \varepsilon)
$$


where the outer solution $U$ has the form

$$
U(x, y ; \varepsilon) \sim \sum_{n=0}^{\infty} \varepsilon^{n} U^{n}(x, y)
$$

while the boundary layer corrections at $x=0$ and $x=1$ have the forms

$$
\tilde{V}(x, y ; \varepsilon) \sim \sum_{n=0}^{\infty} \varepsilon^{n} \tilde{V}^{n}(x, y ; \varepsilon)
$$

and

$$
\tilde{W}(x, y ; \varepsilon) \sim \sum_{n=0}^{\infty} \varepsilon^{n} \tilde{W}^{n}(x, y ; \varepsilon)
$$

respectively. We seek $\tilde{V}^{n}, \tilde{W}^{n}$ for $y>0$ tentatively in the forms

$$
\begin{aligned}
& \tilde{V}^{n}(x, y ; \varepsilon)=\exp \left\{-\frac{\tilde{\sigma}(x, y)}{\varepsilon}\right\} V^{n}(x, y) \\
& \tilde{W}^{n}(x, y ; \varepsilon)=\exp \left\{-\frac{\tilde{\tau}(x, y)}{\varepsilon}\right\} W^{n}(x, y) .
\end{aligned}
$$

Further details on these various functions are given below in this section in the course of their recursive construction.

Substitution of the ansatz (3.1) into (1.1) and (2.1)-(2.2) leads to the determination of $U^{0}$ as the solution of the boundary value problem

$$
\begin{array}{cc}
U_{y y}^{0}=0,(x, y) \in \Omega_{+} ; U^{0}(x, \gamma(x))=\phi(x), & 0 \leqq x \leqq 1 \\
U_{y y}^{0}=U_{x x}^{0},(x, y) \in \Omega_{-} ; U^{0}(x,-x)=\psi(x), & 0 \leqq x \leqq 1 / 2 .
\end{array}
$$

The same DE as in (3.3) is satisfied by $U^{1}$ while for $n \geqq 2$

$$
\begin{aligned}
& U_{y y}^{n}=-U_{x x}^{n-2}, \quad(x, y) \in \Omega_{+} \\
& U_{y y}^{n}=U_{x x}^{n}, \quad(x, y) \in \Omega_{-} .
\end{aligned}
$$

The function $U^{0}$ is the solution of "the reduced problem" obtained by setting $\varepsilon=0$ in (1.1) and by omitting the boundary conditions (2.3)-(2.4).

It is easy to verify in a straightforward way that for smooth $\phi, \psi$ and $\gamma$ the unique solution of (3.3) in the class $\mathscr{C}(\Omega)$ is given by

$$
U^{0}(x, y)=\left\{\begin{array}{l}
\frac{\phi(x)-\chi^{0}(x)}{\gamma(x)} y+\chi^{0}(x), \quad(x, y) \in \bar{\Omega}_{+} \\
\psi\left(\frac{x-y}{2}\right)-\psi\left(\frac{x+y}{2}\right)+\chi^{0}(x+y), \quad(x, y) \in \bar{\Omega}_{-}
\end{array}\right.
$$


where for $x$ in $[0,1]$

$$
\chi^{0}(x):=2 \psi(x / 2)-\psi(0) \exp \left\{-\int_{0}^{x} \frac{d s}{\gamma(s)}\right\}+\int_{0}^{x} \exp \left\{-\int_{s}^{x} \frac{d t}{\gamma(t)}\right\} \frac{\phi(s)-2 \psi(s / 2)}{\gamma(s)} d s
$$

It is observed that the boundary conditions on the subcharacteristics are not necessarily satisfied by $U^{0}$ and this necessitates the introduction of boundary layer corrections $\tilde{V}^{0}$ and $\tilde{W}^{0}$.

Substitution of the ansatz (3.2) into (1.1) and (2.1)-(2.2), and taking into account the $U^{0}$ previously constructed leads to the following boundary value problem for $V^{0}$. In $\Omega_{+}$ this consists of the differential equation

$$
V_{y y}^{0}+\dot{\sigma}^{2}(x) V^{0}=0
$$

subject to the boundary condition

$$
V^{0}(x, \gamma(x))=0
$$

Here $\tilde{\sigma}(x, y)$ must be chosen as a function $\sigma=\sigma(x)$ of $x$ alone in order to balance terms at lowest order and a dot "." indicates $d / d x$ here and below. In $\Omega_{-}$the differential equation for $V^{0}=V$ is

$$
V_{y y}=V_{x x}
$$

subject to the boundary condition

$$
V(x,-x)=0
$$

Considering the boundary value problem for $V^{0}$ we see that we have a somewhat non-standard eigenvalue problem with eigenvalue parameter $\dot{\sigma}^{2}(x)$. We solve this problem in the following way. If $V^{0}(x, 0)=0$, then the ODE eigenvalue problem (3.6), (3.7) in the variable $y$ for $y \geqq 0$ with parameter $x$ has a countable number of eigenvalues given via

$$
\dot{\sigma}(x)=\lambda_{m}(x)=\frac{m \pi}{\gamma(x)}, \quad m=1,2, \ldots
$$

and associated eigenfunctions

$$
\Phi_{m}(x, y)=\sqrt{\frac{2}{\gamma(x)}} \sin \left(\frac{m \pi y}{\gamma(x)}\right)
$$

which have been orthonormalised with respect to an appropriate inner product (for fixed $x$ )

$$
\left\langle\Phi_{m}(x, y), \Phi_{s}(x, y)\right\rangle:=\int_{0}^{\gamma(x)} \Phi_{m}(x, y) \Phi_{s}(x, y) d y=\delta_{m s}
$$


For arbitrary smooth functions $a_{m}^{0}(x)$ (to be determined) the functions

$$
V_{m}^{0}(x, y):=a_{m}^{0}(x) \Phi_{m}(x, y), \quad m=1,2, \ldots
$$

also solve our restricted eigenvalue problem. Since $\widetilde{V}^{0}(x, 0 ; \varepsilon)$ need not vanish we adjoin to the eigenfunctions $V_{m}^{0}$ the function

$$
V_{*}^{0}(x, y: \varepsilon):=A^{o}(x ; \varepsilon)\left[1-\frac{y}{\gamma(x)}\right]
$$

which corresponds to $\dot{\sigma}(x)=0$ and which vanishes for $y=\gamma(x)$ but not $y=0$ and consider for $y \geqq 0$

$$
\tilde{V}^{0}(x, y ; \varepsilon)=V_{*}^{0}(x, y ; \varepsilon)+\sum_{m=1}^{\infty} \exp \left\{-\frac{1}{\varepsilon} \int_{0}^{x} \lambda_{m}(s) d s\right\} V_{m}^{0}(x, y)
$$

with $A^{0}$ to be determined. This expression for $\tilde{V}^{0}$ is a generalisation of the original ansatz assumed above in (3.2).

Using $\widetilde{V}^{0}(x, 0 ; \varepsilon), \tilde{V}_{y}^{0}(x, 0 ; \varepsilon)$ as Cauchy data we solve the Cauchy problem for the wave equation (3.8) in $\Omega_{-}$also subject to the boundary condition (3.9). The flexibility provided by $A^{0}$ allows this seemingly overdetermined problem to be solved in a unique way yielding

$$
\tilde{V}^{0}(x, y ; \varepsilon)=\left\{\begin{array}{l}
A^{0}(x ; \varepsilon)\left[1-\frac{y}{\gamma(x)}\right]+\sum_{m=1}^{\infty} \exp \left\{-\frac{1}{\varepsilon} \int_{0}^{x} \lambda_{m}(s) d s\right\} V_{m}^{0}(x, y), \quad(x, y) \in \bar{\Omega}_{+} \\
A^{0}(x+y ; \varepsilon), \quad(x, y) \in \bar{\Omega}_{-}
\end{array}\right.
$$

where for $x$ in $[0,1]$

$$
A^{0}(x ; \varepsilon):=\sum_{m=1}^{\infty} \int_{1}^{x}\left(\exp \left\{-\int_{s}^{x} \frac{d t}{\gamma(t)}\right\} \exp \left\{-\frac{1}{\varepsilon} \int_{0}^{s} \lambda_{m}(t) d t\right\} V_{m, y}^{0}(s, 0)\right) d s .
$$

Our choice of unity for the lower limit on the integral in (3.11) makes $A^{0}$ exponentially (transcendentally) small away from $x=0$ and of order $O(\varepsilon)$ uniformly in $[0,1]$ (see $(3.18)$ below). Hence the boundary condition (3.9) is satisfied to order $\varepsilon$ and $V_{*}^{0}$ is uniformly $O(\varepsilon)$ on $\Omega$ although it is part of $\tilde{V}^{0}$ which is a zeroth order correction. This term is needed, however, to allow $\tilde{V}^{0}$ to be extended from $\Omega_{+}$to $\Omega_{-}$as an element of $\mathscr{C}(\Omega)$. For the complete specification of $\tilde{V}^{0}$ only the quantities $a_{m}^{0}$ are yet to be determined. Before doing this let us note that similar considerations lead to the determination of $\tilde{W}^{0}$ as

$$
\tilde{W}^{0}(x, y ; \varepsilon)=\left\{\begin{array}{l}
B^{0}(x ; \varepsilon)\left[1-\frac{y}{\gamma(x)}\right]+\sum_{m=1}^{\infty} \exp \left\{-\frac{1}{\varepsilon} \int_{x}^{1} \lambda_{m}(s) d s\right\} W_{m}^{0}(x, y),(x, y) \in \bar{\Omega}_{+} \\
B^{0}(x+y ; \varepsilon), \quad(x, y) \in \bar{\Omega}_{-}
\end{array}\right.
$$


where $W_{m}^{0}(x, y)=b_{m}^{0}(x) \Phi_{m}(x, y)$ with $b_{m}^{0}$ to be determined and for $x \in[0,1]$

$$
B^{0}(x, \varepsilon):=\sum_{m=1}^{\infty} \int_{0}^{x} \exp \left\{-\frac{1}{\varepsilon} \int_{s}^{1} \lambda_{m}(t) d t\right\} \exp \left\{-\int_{s}^{x} \frac{d t}{\gamma(t)}\right\} W_{m, y}^{0}(s, 0) d s
$$

Thus $\tilde{W}^{0}$ is exponentially small away from $\Gamma_{1}$.

These results make it necessary to modify our ansatz for $\tilde{V}^{n}$ and $\tilde{W}^{n}$ in $y>0$ corresponding to the forms of $\tilde{V}^{0}$ and $\tilde{W}^{0}$ above. Further experimenting leads us to take

$$
\tilde{V}^{n}(x, y ; \varepsilon)=\left\{\begin{array}{l}
V_{*}^{n}(x, y ; \varepsilon)+\sum_{m=1}^{\infty} \exp \left\{-\frac{1}{\varepsilon} \int_{0}^{x} \lambda_{m}(s) d s\right\} V_{m}^{n}(x, y), \quad(x, y) \in \bar{\Omega}_{+} \\
V_{*}^{n}(x+y, 0 ; \varepsilon), \quad(x, y) \in \bar{\Omega}_{-}
\end{array}\right.
$$

where for $y \geqq 0$

$$
V_{*}^{n}(x, y ; \varepsilon)=\sum_{m=1}^{\infty} \int_{1}^{x} \exp \left\{-\frac{1}{\varepsilon} \int_{0}^{s} \lambda_{m}(t) d t\right\} \delta_{m}^{n}(s, x, y) d s
$$

with $V_{m}^{n}$ and $\delta_{m}^{n}$ to be determined. The revised form of $W^{n}$ is similar

$$
\tilde{W}^{n}(x, y ; \varepsilon)=\left\{\begin{array}{l}
W_{*}^{n}(x, y ; \varepsilon)+\sum_{m=1}^{\infty} \exp \left\{-\frac{1}{\varepsilon} \int_{x}^{1} \lambda_{m}(s) d s\right\} W_{m}^{n}(x, y), \quad(x, y) \in \bar{\Omega}_{+} \\
W_{*}^{n}(x+y, 0 ; \varepsilon), \quad(x, y) \in \bar{\Omega}_{-}
\end{array}\right.
$$

where for $y \geqq 0$

$$
W_{*}^{n}(x, y ; \varepsilon):=\sum_{m=1}^{\infty} \int_{0}^{x} \exp \left\{-\frac{1}{\varepsilon} \int_{s}^{1} \lambda_{m}(t) d t\right\} \varepsilon_{m}^{n}(s, x, y) d s
$$

with $W_{m}^{n}$ and $\varepsilon_{m}^{n}$ to be determined.

The modified ansatz does not change the problem for $\tilde{V}^{0}$ or its solution (3.10) but it does lead to the following modified problem for $V_{m}^{1}$ for $y>0$

$$
\begin{aligned}
V_{m, y y}^{1}+\lambda_{m}^{2}(x) V_{m}^{1} & =2 \lambda_{m}(x) V_{m, x}^{0}+\lambda_{m}(x) V_{m}^{0}+\lambda_{m}(x)\left(1-\frac{y}{\gamma(x)}\right) V_{m, y}^{0}(x, 0) \\
& =: f_{m}^{1}(x, y) \\
V_{m}^{1}(x, 0) & =V_{m}^{1}(x, \gamma(x))=0 .
\end{aligned}
$$


The problem for $V_{m}^{n}$ for $n \geqq 2$ then consists of the equation in $\Omega_{+}$

$$
\begin{aligned}
V_{m, y y}^{n}+\lambda_{m}^{2}(x) V_{m}^{n}= & {\left[2 \lambda_{m}(x) V_{m, x}^{n-1}+\lambda_{m}(x) V_{m}^{n-1}-V_{m, x x}^{n-2}\right.} \\
& +\lambda_{m}(x) \delta_{m}^{n-1}(x, x, y)-D_{1} \delta_{m(x, x, y)}^{n-2}-2 D_{2} \delta_{m}^{n-2}(x, x, y) \\
= & : f_{m}^{n}(x, y)
\end{aligned}
$$

with the same boundary condition as in (3.14). In (3.15) and hereafter $D_{i}$, as usual, denotes differentiation with respect to the $i$ th argument. The $\delta_{m}^{n}$ are to satisfy

$$
\delta_{m, y y}^{1}=0 ; \delta_{m, y y}^{n}=-\delta_{m, x x}^{n-2} \text { for } n \geqq 2, \quad(x, y) \in \Omega_{+}
$$

with the boundary conditions

$$
\begin{gathered}
\delta_{m}^{n}(s, x, \gamma(x))=0 \\
\delta_{m}^{n}(x, x, 0)=V_{m, y}^{n}(x, 0), \quad D_{2} \delta_{m}^{n}(s, x, 0)=D_{3} \delta_{m}^{n}(s, x, 0) .
\end{gathered}
$$

The latter conditions ensure a $C^{1}$ continuation of $\tilde{V}^{n}$ to $y<0$ as a solution of the wave equation (3.8). Observe that for $n=0,1$

$$
\delta_{m}^{n}(s, x, y)=\left(1-\frac{y}{\gamma(x)}\right) \exp \left\{-\int_{s}^{x} \frac{d t}{\gamma(t)}\right\} V_{m, y}^{n}(s, 0) .
$$

Now we return to the determination of the $a_{m}^{0}(x)$. A necessary and sufficient condition for the existence of a solution to the problem (3.14) is the orthogonality condition

$$
\left\langle\Phi_{m}(x, y), f_{m}^{1}(x, y)\right\rangle=0
$$

which yields the ODE

$$
\dot{a}_{m}^{0}+\left[\frac{\lambda_{m}(x)}{2 \lambda_{m}(x)}+\frac{1}{\gamma(x)}\right] a_{m}^{0}=0
$$

with solution

$$
a_{m}^{0}(x)=\sqrt{\frac{\gamma(x)}{\gamma(0)}} \exp \left\{-\int_{0}^{x} \frac{d t}{\gamma(t)}\right\} a_{m}^{0}(0)
$$

determined uniquely by $a_{m}^{0}(0)$. The latter are chosen to meet the boundary condition on $x=0$

$$
\phi_{0}(y)-U^{0}(0, y)=\tilde{V}(0, y ; \varepsilon)=\sum_{m=1}^{\infty} a_{m}^{0}(0) \Phi_{m}(0, y)
$$


so that $a_{m}^{0}(0)$ is the Fourier coefficient

$$
a_{m}^{0}(0)=\left\langle\phi_{0}(y)-U^{0}(0, y), \Phi_{m}(0, y)\right\rangle
$$

Note that the function $\phi_{0}(y)-U^{0}(0, y)$ vanishes at $y=0$ and $y=\gamma(0)$ due to the compatibility conditions $\phi_{0}(0)=\psi(0)$ and $\phi_{0}(\gamma(0))=\phi(0)$ respectively. Thus this function has a continuous periodic extension to all of $\mathbb{R}$. Further discussion of the resulting Fourier series appears in Section 5 . Now $\tilde{V}^{0}$ is completely determined.

In a similar way one finds for $\tilde{W}^{0}$ in (3.12)

$$
b_{m}^{o}(x)=\sqrt{\frac{\gamma(x)}{\gamma(1)}} \exp \left\{-\int_{1}^{x} \frac{d t}{\gamma(t)}\right\} b_{m}^{o}(1)
$$

and $b_{m}^{o}(1)$ is chosen so that $\tilde{W}^{0}(1, y ; \varepsilon)=\phi_{1}(y)-U^{0}(1, y)$, i.e.

$$
b_{m}^{0}(1)=\left\langle\phi_{1}(y)-U^{0}(1, y), \Phi_{m}(1, y)\right\rangle .
$$

Note here that the function $\phi_{1}(y)-U^{0}(1, y)$, vanishes at $y=\gamma(1)$ due to the compatibility condition $\phi_{1}(\gamma(1))=\phi(1)$ but does not necessarily vanish for $y=0$. Thus the Fourier series for $\phi_{1}(y)-U^{0}(1, y)$ will not have this function as its pointwise limit unless the rather restrictive condition that $\phi_{1}(0)=U^{0}(1,0)$ is imposed. Further discussion on this point is given in Section 5 . With this $\tilde{W}^{0}$ is completely determined.

In assessing the accuracy of the lowest order approximation $\tilde{U}^{0}+\tilde{V}^{0}+\tilde{W}^{0}$, orders of magnitude of the difference between $\tilde{U}^{0}+\tilde{V}^{0}+\tilde{W}^{0}$ along the boundary and the prescribed data must be calculated. To this end, we note that from earlier results

$$
A^{0}(x ; \varepsilon)=\varepsilon \sqrt{\frac{2}{\gamma(0)}} \exp \left\{-\int_{0}^{x} \frac{d t}{\gamma(t)}\right\} \sum_{m=1}^{\infty} a_{m}^{0}(0)\left[\exp \left\{-\frac{m \pi}{\varepsilon} \int_{0}^{1} \frac{d t}{\gamma(t)}\right\}-\exp \left\{-\frac{m \pi}{\varepsilon} \int_{0}^{x} \frac{d t}{\gamma(t)}\right\}\right]
$$

Consider now $\Gamma_{0}$. From (3.10)

$$
\begin{aligned}
\tilde{V}(0, y ; \varepsilon) & =A^{0}(0 ; \varepsilon)\left[1-\frac{y}{\gamma(0)}\right]+\sum_{m=1}^{\infty} V_{m}^{0}(0, y) \\
& =A^{0}(0 ; \varepsilon)\left[1-\frac{y}{\gamma(0)}\right]+\sum_{m=1}^{\infty} a_{m}^{0}(0) \Phi_{m}(0, y) .
\end{aligned}
$$

By construction, the infinite sum is precisely $\phi_{0}(y)-U^{0}(0, y)$ (using $\phi_{0}(y)-U^{0}(0, y)=0$ at $y=0, \gamma(0))$.

Thus

$$
\begin{aligned}
\widetilde{V}(0, y ; \varepsilon)+U^{0}(0, y)-\phi_{0}(y) & =A^{0}(0 ; \varepsilon)\left[1-\frac{y}{\gamma(0)}\right] \\
& =\varepsilon \alpha^{0}\left[1-\frac{y}{\gamma(0)}\right]+E(\varepsilon)
\end{aligned}
$$


by (3.18), where

$$
\alpha^{0}=\sqrt{\frac{2}{\gamma(0)}} \sum_{m=1}^{\infty} a_{m}^{0}(0)
$$

and $E(\varepsilon)$ denotes terms transcendentally small in $\varepsilon$. Similarly on the boundary $C_{0}$, using the condition $U^{0}(x,-x)=\psi(x)$,

$$
\begin{aligned}
\tilde{V}^{0}(x,-x ; \varepsilon)+U^{0}(x,-x)-\psi(x) & =A(0 ; \varepsilon) \\
& =-\varepsilon \alpha^{0}+E(\varepsilon) .
\end{aligned}
$$

Provided that $\phi_{1}$ meets certain conditions (see Section 5), an analogous calculation for $\tilde{W}^{0}$ shows that along $\Gamma_{1}$,

$$
\begin{aligned}
W^{0}(1, y ; \varepsilon)+U^{0}(1, y)-\phi_{1}(y) & =B^{0}(1 ; \varepsilon)\left[1-\frac{y}{\gamma(1)}\right] \\
& =\varepsilon \beta^{0}\left[1-\frac{y}{\gamma(1)}\right]+E(\varepsilon)
\end{aligned}
$$

where

$$
\beta^{0}=\frac{2}{\gamma(1)} \sum_{m=1}^{\infty} b_{m}^{0}(1)
$$

Finally noting that $\tilde{V}^{0}$ and $\tilde{W}^{0}$ are transcendentally small in $\varepsilon$ away from $\Gamma_{0}$ and $\Gamma_{1}$ respectively, it follows that $\tilde{U}^{0}+\tilde{V}^{0}+\tilde{W}^{0}$ formally satisfies the differential equation (1.1) exactly in $\Omega_{-}$and to order $\varepsilon$ in $\Omega_{+}$while the boundary condition (2.2) is satisfied exactly and the boundary conditions (2.1), (2.3) and (2.4) are satisfied to order $\varepsilon$. We proceed to the construction of $\tilde{U}^{1}, \tilde{V}^{1}$ and $\tilde{W}^{1}$ in order to solve our boundary value problem to order $\varepsilon^{2}$. In particular $U^{1}$ is to be chosen so that the $O(\varepsilon)$ terms in (3.19) and (3.20) are cancelled.

As observed above $U^{1}$ must satisfy the same differential equations as for $U^{0}$ in (3.3) but the boundary conditions are $U^{1}(x, \gamma(x))=0$ and $U^{1}(x,-x)=\alpha^{0}$. Thus

$$
U^{1}(x, y):=\left\{\begin{array}{l}
\alpha^{0}\left[1-\frac{y}{\gamma(x)}\right] \exp \left\{-\int_{0}^{x} \frac{d t}{\gamma(t)}\right\} \\
\alpha^{0} \exp \left\{-\int_{0}^{x+y} \frac{d t}{\gamma(t)}\right\}
\end{array}\right.
$$

is the unique element of $\mathscr{C}(\Omega)$ satisfying these conditions. Now $\tilde{V}^{1}$ and $\tilde{W}^{1}$ are to be constructed to provide corrections to the boundary conditions on $\Gamma_{0}$ and $\Gamma_{1}$.

As a result of fulfilling the orthogonality condition (3.17) the problem (3.14) is 
solvable with general solution

$$
V_{m}^{1}(x, y)=v_{m}^{1}(x, y)+a_{m}^{1}(x) \Phi_{m}(x, y)
$$

where

$$
v_{m}^{1}(x, y)=\sum_{\substack{s=1 \\ s \neq m}}^{\infty} \frac{\left\langle f_{m}^{1}(x, y), \Phi_{s}(x, y)\right\rangle}{\lambda_{m}^{2}(x)-\lambda_{s}^{2}(x)} \Phi_{s}(x, y)
$$

and the $a_{m}^{1}$ are arbitrary. With this $V_{m}^{1}$ we obtain $\delta_{m}^{1}$ from (3.16) to get $V_{*}^{1}$ and form for $y \geqq 0$

$$
\tilde{V}^{1}(x, y ; \varepsilon)=V_{*}^{1}(x, y ; \varepsilon)+\sum_{m=1}^{\infty} \exp \left\{-\frac{1}{\varepsilon} \int_{0}^{x} \lambda_{m}(s) d s\right\} V_{m}^{1}(x, y) .
$$

As we did for $\widetilde{V}^{0}$ we use $\widetilde{V}^{1}(x, 0 ; \varepsilon), \widetilde{V}_{y}^{1}(x, 0 ; \varepsilon)$ as Cauchy data for the Cauchy problem for (3.8) subject to (3.9) (to order one) to obtain for $y \leqq 0$

$$
\widetilde{V}^{1}(x, y ; \varepsilon)=A^{1}(x+y ; \varepsilon), \quad(x, y) \in \bar{\Omega}_{-}
$$

where for $x$ in $[0,1]$

$$
A^{1}(x ; \varepsilon):=\sum_{m=1}^{\infty} \int_{1}^{x}\left(\exp \left\{-\int_{s}^{x} \frac{d t}{\gamma(t)}\right\} \exp \left\{-\frac{1}{\varepsilon} \int_{0}^{s} \lambda_{m}(t) d t\right\} V_{m, y}^{1}(s, 0)\right) d s .
$$

Now $a_{m}^{1}$ is determined from the orthogonality condition

$$
\left\langle\Phi_{m}(x, y), f_{m}^{2}(x, y)\right\rangle=0
$$

leading to

$$
a_{m}^{1}(x)=\sqrt{\frac{\gamma(x)}{\gamma(0)}} \exp \left\{-\int_{0}^{x} \frac{d t}{\gamma(t)}\right\} a_{m}^{1}(0)+\sqrt{\gamma(x)} \int_{0}^{x}[\gamma(s)]^{-1 / 2} \exp \left\{-\int_{s}^{x} \frac{d t}{\gamma(t)}\right\} A_{m}^{1}(s) d s
$$

where

$$
\begin{aligned}
A_{m}^{1}(x):= & \frac{1}{2} \lambda_{m}^{-1}(x)\left[\ddot{a}_{m}^{0}(x)+a_{m}^{0}(x)\left\langle\Phi_{m, x x}(x, y), \Phi_{m}(x, y)\right\rangle\right. \\
& +\left\langle D_{1} \delta_{m}^{0}(x, x, y)+2 D_{2} \delta_{m}^{0}(x, x, y), \Phi_{m}(x, y)\right\rangle \\
& \left.-\sqrt{\frac{2}{\gamma(x)}} v_{m, y}^{1}(x, 0)\right]-\left\langle v_{m, x}^{1}(x, y), \Phi_{m}(x, y)\right\rangle
\end{aligned}
$$

and the $a_{m}^{1}(0)$ are chosen so that

$$
0=\tilde{V}^{1}(0, y ; \varepsilon)=\sum_{m=1}^{\infty}\left[v_{m}^{1}(0, y)+a_{m}^{1}(0) \Phi_{m}(0, y)\right]
$$


LAVRENT'EV-BITSADZE EQUATION

59

and hence

$$
a_{m}^{1}(0)=\sum_{\substack{s=1 \\ s \neq m}}^{\infty} \frac{\left\langle f_{s}^{1}(0, y), \Phi_{m}(0, y)\right\rangle}{\lambda_{m}^{2}(0)-\lambda_{s}^{2}(0)}
$$

thus determining $\widetilde{V}^{1}$ completely.

The function $\widetilde{W}^{1}$ is constructed in an entirely analogous manner with the result

$$
\tilde{W}^{1}(x, y ; \varepsilon)=\left\{\begin{array}{l}
B^{1}(x ; \varepsilon)\left[1-\frac{y}{\gamma(x)}\right]+\sum_{m=1}^{\infty} \exp \left\{-\frac{1}{\varepsilon} \int_{x}^{1} \lambda_{m}(s) d s\right\} W_{m}^{1}(x, y), \quad(x, y) \in \Omega_{+} \\
B^{1}(x+y ; \varepsilon), \quad(x, y) \in \Omega_{-}
\end{array}\right.
$$

where for $x$ in $[0,1]$

$$
B^{1}(x ; \varepsilon)=\sum_{m=1}^{\infty} \int_{0}^{x} \exp \left\{-\int_{s}^{x} \frac{d t}{\gamma(t)}\right\} \exp \left\{-\frac{1}{\varepsilon} \int_{s}^{1} \lambda_{m}(t) d t\right\} W_{m, y}^{1}(s, 0) d s .
$$

Further we have

$$
\begin{gathered}
W_{m}^{1}(x, y)=\omega_{m}^{1}(x, y)+b_{m}^{1}(x) \Phi_{m}(x, y), \\
\omega_{m}^{1}(x, y)=\sum_{\substack{s=1 \\
s \neq m}}^{\infty} \frac{\left\langle g_{m}^{1}(x, y), \Phi_{s}(x, y)\right\rangle}{\lambda_{m}^{2}(x)-\lambda_{s}^{2}(x)} \Phi_{s}(x, y) \\
g_{m}^{1}(x, y)=-2 \lambda_{m}(x) W_{m, x}^{0}-\lambda_{m}(x) W_{m}^{0}-\lambda_{m}(x)\left(1-\frac{y}{\gamma(x)}\right) W_{m, y}^{0}(x, 0), \\
b_{m}^{1}(x)=\sqrt{\frac{\gamma(x)}{\gamma(1)}} \exp \left\{-\int_{1}^{x} \frac{d t}{\gamma(t)}\right\} b_{m}^{1}(1)-\sqrt{\gamma(x)} \int_{x}^{1}[\gamma(s)]^{-1 / 2} \exp \left\{-\int_{s}^{x} \frac{d t}{\gamma(t)}\right\} B_{m}^{1}(s) d s, \\
B_{m}^{1}(x)=(-1 / 2) \lambda_{m}^{-1}(x)\left\{\ddot{b}_{m}^{0}(x)+b_{m}^{0}(x)\left\langle\Phi_{m, x x}(x, y), \Phi_{m}(x, y)\right\rangle\right. \\
+\left\langle D_{1} \varepsilon_{m}^{0}(x, x, y)+2 D_{2} \varepsilon_{m}^{0}(x, x, y), \Phi_{m}(x, y)\right\rangle \\
\left.+\sqrt{\frac{2}{\gamma(x)}} \omega_{m, y}^{1}(x, 0)\right\}-\left\langle\omega_{m, x}^{1}(x, y), \Phi_{m}(x, y)\right\rangle \\
\varepsilon_{m}^{n}(s, x, y)=\left[1-\frac{y}{\gamma(x)}\right] \exp \left\{-\int_{s}^{x} \frac{d t}{\gamma(t)}\right\} W_{m, y}^{n}(s, 0), \quad n=0,1
\end{gathered}
$$

https://doi.org/10.1017/S0013091500028066 Published online by Cambridge University Press 
and

$$
b_{m}^{1}(1)=\sum_{\substack{s=1 \\ s \neq m}}^{\infty} \frac{\left\langle g_{s}^{1}(1, y), \Phi_{m}(1, y)\right\rangle}{\lambda_{m}^{2}(1)-\lambda_{s}^{2}(1)}-\left\langle\Phi_{m}(1, y), U^{1}(1, y)\right\rangle
$$

For $n \geqq 2$ the procedure continues in a similar way allowing recursive determination of the $U^{n}, \tilde{V}^{n}$ and $\tilde{W}^{n}$ for all $n$.

\section{An a priori estimate}

For the proof of uniform asymptotic validity of the expansions constructed in the previous section we use in Section 5 the simple a priori estimate given below in Lemma 2. Its proof is based upon

Lemma 1. (Comparison Theorem) Let $v$ be in the class $\mathscr{C}(\Omega)$ and suppose that

$$
\begin{cases}\varepsilon^{2} v_{x x}+v_{y y} \geqq 0 & \text { in } \Omega_{+} \\ v_{x x}-v_{y y}=0 & \text { in } \Omega_{-}\end{cases}
$$

with

$$
\left\{\begin{array}{l}
v \leqq 0 \text { on } \Gamma_{0} \cup \Gamma \cup \Gamma_{1} \\
v=v(0,0 ; \varepsilon) \text { on } C_{0}
\end{array}\right.
$$

Then $v \leqq 0$ in $\Omega$.

Proof. The differential equation in $\Omega_{-}$and the boundary condition on $C_{0}$ imply that in $\Omega_{-}$

$$
v(x, y ; \varepsilon)=g(x+y ; \varepsilon)-g(0 ; \varepsilon)+v(0,0 ; \varepsilon)
$$

for a $C^{2}(0,1) \cap C[0,1]$ function $g$ and hence

$$
\max _{\bar{\Omega}_{-}} V=\max _{0 \leqq x \leqq 1} v(x, 0 ; \varepsilon)
$$

so that

$$
\max _{\overline{\mathbf{\Omega}}} v=\max _{\overline{\mathbf{\Omega}}_{+}} v
$$

But this maximum does not occur at $\left(x_{0}, 0\right.$ for $0<x_{0}<1$ since at such a point

$$
v_{x}\left(x_{0}, 0 ; \varepsilon\right)=0
$$


and so from (4.1)

$$
v_{y}\left(x_{0}, 0 ; \varepsilon\right)=0
$$

in violation of Hopf's [7] boundary point principle. Now from the maximum principle for elliptic inequalities

$$
\max _{\overline{\mathbf{\Omega}}_{+}} v=\max _{\Gamma_{0} \cup \Gamma \cup \Gamma_{1}} v \leqq 0
$$

the assertion follows.

Remark. As is clear from the proof, Lemma 1 is a slight variant of a maximum principle for equation (1.1) (cf. [1; p. 74]).

From the Comparison Theorem one obtains in a familiar way

Lemma 2. (a priori Estimate) Let $F$ belong to $C\left(\bar{\Omega}_{+}\right)$and let $f$ belong to $C\left(\Gamma_{0} \cup \Gamma \cup \Gamma_{1}\right)$. Suppose that $w$ is a function in the class $\mathscr{C}(\Omega)$ such that

$$
\left\{\begin{array}{l}
\varepsilon^{2} w_{x x}+w_{y y}=F \quad \text { in } \Omega_{+} \\
w_{x x}-w_{y y}=0 \quad \text { in } \Omega_{-}
\end{array}\right.
$$

and

$$
\begin{cases}w=f & \text { on } \Gamma_{0} \cup \Gamma \cup \Gamma_{1} \\ w=f(0,0) & \text { on } C_{0} .\end{cases}
$$

Then there exists a constant $M$, independent of $\varepsilon$ as well as $(x, y)$, such that

$$
|w(x, y ; \varepsilon)| \leqq M\|F\|+\|f\|
$$

where the norms are the maximum norms over $\bar{\Omega}_{+}$and $\Gamma_{0} \cup \Gamma \cup \Gamma_{1}$ respectively.

Proof. Let $h=h(\xi)$ be any real-valued $C^{2}$ function of the real variable $\xi$ such that, for given $\xi_{0}=\max (x+y)$ for $(x, y)$ in $\Omega, h^{\prime \prime}(\xi) \leqq-1$ and $h(\xi) \geqq 0$ for $0 \leqq \xi \leqq \xi_{0}$. Then the function $v$

$$
v= \pm w-h(x+y)\|F\|-\|f\|
$$

satisfies the hypotheses of Lemma 1 and so the assertion follows with $M=\max h$ on $\left[0, \xi_{0}\right]$.

\section{Justification}

Before stating our main result we analyze briefly the results of the formal construction of the previous section. As is clear from (3.4) the function $U^{0}$ belongs to $\mathscr{C}(\Omega)$ under the 
sufficient conditions that $\phi, \gamma$ belong to $C^{2}[0,1]$ and $\psi$ belongs to $C^{2}\left[0, \frac{1}{2}\right]$. Under the additional conditions stated in the theorem below the correction term $\tilde{V}^{0}$ also belongs to $\mathscr{C}(\Omega)$. A main factor in the validity of this assertion is that the compatibility conditions $\phi_{0}(0)=\psi(0)$ and $\phi_{0}\left(\gamma_{0}\right)=\phi(0)$ ensure a continuous periodic extension of $\phi_{0}(y)$ $-U^{0}(0, y)$ to the entire real line.

The correction term $\tilde{W}^{0}$, however, poses difficulties with regard to the convergence of the corresponding series. The compatibility condition $\phi_{1}\left(\gamma_{1}\right)=\phi(1)$ guarantees that $\phi_{1}(y)$ $-U^{0}(1, y)$ vanishes at $y=\gamma_{1}$ but this function need not vanish at $y=0$ and so the corresponding Fourier series will not converge to the function at the end points of the interval of concern.

At this point it becomes necessary to restrict $\phi_{1}(y)$, the boundary data on $x=1$, to be such that $\phi_{1}(y)-U^{0}(1, y)$ vanishes when $y=0$. Of course, by this assumption we may be avoiding the interesting question of corner expansions and corner singularities which occur for equations of mixed type. In contrast to the case for PDE's of unchanging type the topic of corner expansions in the present case is unexplored and remains a subject for future study.

A difficulty similar to that for $\tilde{W}^{0}$ arises in the convergence of the series representation of $\tilde{W}^{1}$ unless rather special and overly restrictive conditions are imposed. Since this may involve the suppression of corner expansions which would otherwise be present, our theorem gives uniform results only up to order $O(\varepsilon)$. There is no such difficulty for $V^{1}$ and it is considered worthwhile to indicate the nature of the estimates involved to establish convergence of the series. These are presented in Appendix B.

We now state our main result which is embodied in the

Theorem. Let $\phi, \gamma \in C^{2}[0,1], \psi \in C^{2}[0,1 / 2]$ and for $i=0,1, \phi_{i} \in C^{4}\left[0, \gamma_{i}\right]$ with the usual compatibility conditions for continuity of the boundary data:

$$
\psi(0)=\phi_{0}(0), \quad \phi(0)=\phi_{0}\left(\gamma_{0}\right), \quad \phi(1)=\phi_{1}\left(\gamma_{1}\right)
$$

Suppose in addition that $D^{2 j} \phi_{i}(0)=D^{2 j} \phi_{i}\left(\gamma_{i}\right)=0$ for $j=1,2$ and

$$
\phi_{1}(0)=U^{0}(1,0)
$$

Then

$$
u(x, y ; \varepsilon)=U^{0}(x, y)+\tilde{V}^{0}(x, y ; \varepsilon)+\tilde{W}^{0}(x, y ; \varepsilon)+O(\varepsilon)
$$

uniformly on $\bar{\Omega}$.

Remark. Under the hypotheses on the data an elementary computation shows that both $a_{m}^{0}(0)$ and $b_{m}^{0}(0)$ are $O\left(m^{-4}\right)$ as $m \rightarrow \infty$. Hence it follows from Appendix $A$ that the term-wise differentiated series (up through the second order) for $\tilde{V}^{0}$, and $\tilde{W}^{0}$ converge uniformly on $\bar{\Omega}_{+}$and $\bar{\Omega}_{-}$separately. Thus each of these functions belongs to $C^{2}\left(\bar{\Omega}_{+}\right) \cap C^{2}\left(\bar{\Omega}_{-}\right)$. Then the matching for smoothness along $y=0$ in their construction ensures that they belong to $\mathscr{C}(\Omega)$. 
Proof. Introduce the function

$$
Z(x, y ; \varepsilon):=u(x, y ; \varepsilon)-\left[U^{0}(x, y)+\tilde{V}^{0}(x, y ; \varepsilon)+\tilde{W}^{0}(x, y ; \varepsilon)\right]
$$

which by our remark belongs to $\mathscr{C}(\Omega)$.

It is straightforward to verify directly from the definitions that $U^{0}, \tilde{V}^{0}$, and $\tilde{W}^{0}$ each satisfy (1.1) in $\Omega_{-}$and hence the same is true for $Z$. Moreover by direct computation

$$
\varepsilon^{2} Z_{x x}+Z_{y y}=O(\varepsilon)
$$

uniformly in $\Omega_{+}$and

$$
\begin{aligned}
& \left.Z\right|_{\Gamma}=0 \\
& \left.Z\right|_{C_{0}}=-A^{0}(0 ; \varepsilon)=O(\varepsilon) \\
& \left.Z\right|_{\Gamma_{0}}=-\tilde{W}^{0}(0, y ; \varepsilon)-A^{0}(0 ; \varepsilon)\left[1-\frac{y}{\gamma(0)}\right]=O(\varepsilon) \\
& \left.Z\right|_{\Gamma_{1}}=-\tilde{V}^{0}(1, y ; \varepsilon)-B^{0}(1 ; \varepsilon)\left[1-\frac{y}{\gamma(1)}\right]=O(\varepsilon)
\end{aligned}
$$

uniformly on their respective segments. Then $Z$ satisfies the hypotheses for the function $w$ in Lemma 2 of Section 4 with $F=O(\varepsilon)$ uniformly on $\Omega_{+}$and $f=O(\varepsilon)$ uniformly on $\Gamma_{0} \cup \Gamma \cup \Gamma_{1}$ with $f(0,0)=-A^{0}(0 ; \varepsilon)$. From this the assertion of the theorem follows.

Remark. Because $A^{0}$ and $B^{0}$ are uniformly $O(\varepsilon)$ on $[0,1]$ it follows from the theorem that

$$
u=U^{0}+\bar{\nabla}^{0}+\bar{W}^{0}+O(\varepsilon)
$$

where the bar indicates that the terms involving $A^{0}$ and $B^{0}$ have been omitted from $\tilde{V}^{0}$ and $\tilde{W}^{0}$ respectively. The omitted terms are needed for the solutions to be in the class $\mathscr{C}(\Omega)$. In this connection it is interesting to note that the $O(1)$ boundary layer correction $\tilde{V}^{0}$ on $\Gamma_{0}$ carries over into the hyperbolic region as an $O(\varepsilon)$ boundary layer on the characteristic $C_{0}$.

\section{Appendix A: Convergence of the series}

In this appendix we consider the convergence of the series constructed in Section 3 for smooth and suitably restricted data. It does not seem possible to avoid the tedious nature of the required estimates and hence we restrict ourselves to giving details (see Appendix B) only for terms up to order $\varepsilon^{2}$. Higher order terms require smoother data and further restrictions for the estimates to be valid.

We begin with the series for $\tilde{V}^{0}$ which is defined in (3.10) as a sum of two series, the 
second appearing in the definition of $A^{0}$. It is easy to see that each of these series and the term-wise differentiated series up through the second order (with respect to $x$ and/or $y$ ) has the following majorant for each positive $\varepsilon$

$$
C \sum_{m=1}^{\infty}\left|a_{m}^{0}(0)\right| \lambda_{m}^{2}(0)
$$

uniformly on $\bar{\Omega}$. The constant $C$ may depend on $\varepsilon$. Thus from (A.1) $\tilde{V}^{o}$ belongs to $C^{2}$ in $\bar{\Omega}_{+}$and $\bar{\Omega}_{-}$separately provided

$$
a_{m}^{0}(0)=O\left(m^{-\alpha}\right), \quad m \rightarrow \infty
$$

for $\alpha>3$. Under this assumption the matching for smoothness in the construction of $\tilde{V}^{0}$ then ensures that $\tilde{V}^{0}$ belongs to $\mathscr{C}(\Omega)$. Similarly, under the assumption that

$$
b_{m}^{0}(1)=O\left(m^{-\beta}\right), \quad m \rightarrow \infty
$$

for $\beta>3$, an identical proof guarantees that $\tilde{W}^{0}$ belongs to $\mathscr{C}(\Omega)$.

The series for $\widetilde{V}^{1}$ are not quite so easily handled since the estimate is not trivial as is (A.1) in the case of $\tilde{V}^{0}$. The key estimate is supplied by (B.1) in Appendix B. Using the integral comparison test for single and double series one sees that the series in (B.1) converges provided

$$
\left|a_{m}^{0}(0)\right|=O\left(m^{-a}\right)
$$

with $\alpha>6$. Now the argument proceeds as for $\tilde{V}^{0}$ to obtain that $\tilde{V}^{1}$ belongs to $\mathscr{C}(\Omega)$ provided that the additional conditions that $\phi_{0} \in C^{7}\left[0, \gamma_{0}\right]$ and that $D^{6} \phi_{0}(0)=D^{6} \phi_{0}\left(\gamma_{0}\right)$ $=0$ are met. The function $\tilde{W}^{1}$ may be treated in the same way but only by introducing an auxiliary problem to eliminate otherwise restrictive conditions on the data.

\section{Appendix B: Some estimates}

The following estimates may be obtained by straightforward computation. We use the symbol $C$ for a generic constant which may differ in the various inequalities but is always independent of $m$ and $s$ as well as $x$ and $y$. By $\lambda_{m}$ we denote $\lambda_{m}(x)$ at any fixed but convenient point, say for definiteness at $x=0$.

The key estimate (B.1) appears at the end of the appendix.

$$
\begin{aligned}
& \left|\left\langle\Phi_{m}(x, y), \Phi_{s, x}(x, y)\right\rangle\right| \leqq C \frac{\lambda_{m} \lambda_{s}}{\left|\lambda_{m}^{2}-\lambda_{s}^{2}\right|} \\
& \left|\left\langle\Phi_{m}(x, y), \Phi_{s, x x}(x, y)\right\rangle\right| \leqq C \frac{\lambda_{s} \lambda_{m}}{\left|\lambda_{m}^{2}-\lambda_{s}^{2}\right|}\left[1+\frac{\lambda_{s}^{2}}{\left|\lambda_{m}^{2}-\lambda_{s}^{2}\right|}\right] \\
& \left|\left\langle\Phi_{m}(x, y), \Phi_{s, x x x}(x, y)\right\rangle\right| \leqq C \frac{\lambda_{m} \lambda_{s}^{3}}{\left|\lambda_{m}^{2}-\lambda_{s}^{2}\right|}\left[1+\frac{\lambda_{m}^{2}+\lambda_{s}^{2}}{\left|\lambda_{m}^{2}-\lambda_{s}^{2}\right|}\right]
\end{aligned}
$$




$$
\begin{aligned}
& \left|\left\langle\Phi_{m}(x, y), \Phi_{m, x x}(x, y)\right\rangle\right| \leqq C \lambda_{m}^{2} \\
& \left|\left\langle\Phi_{m}(x, y), \Phi_{m, x x x}(x, y)\right\rangle\right| \leqq C \lambda_{m}^{2} \\
& \left|\left\langle\Phi_{m, x}(x, y), \Phi_{s, x}(x, y)\right\rangle\right| \leqq C \frac{\lambda_{s} \lambda_{m}}{\left|\lambda_{m}^{2}-\lambda_{s}^{2}\right|}\left[1+\frac{\lambda_{m}^{2}+\lambda_{s}^{2}}{\left|\lambda_{m}^{2}-\lambda_{s}^{2}\right|}\right] \\
& \left|\left\langle\Phi_{m, x}(x, y), \Phi_{s, x x}(x, y)\right\rangle\right| \leqq C \frac{\lambda_{s} \lambda_{m}}{\left|\lambda_{m}^{2}-\lambda_{s}^{2}\right|}\left[\lambda_{m} \lambda_{s}+\frac{\lambda_{m}^{2}+\lambda_{s}^{2}}{\left|\lambda_{m}^{2}-\lambda_{s}^{2}\right|}+\frac{\lambda_{s} \lambda_{m}\left(\lambda_{m}^{2}+\lambda_{s}^{2}\right)}{\left|\lambda_{m}^{2}-\lambda_{s}^{2}\right|^{2}}\right] \\
& \left|\left\langle\Phi_{m, x}(x, y), \Phi_{m, x x}(x, y)\right\rangle\right| \leqq C \lambda_{m}^{2} \\
& \left|\left\langle\Phi_{m}(x, y), v_{m, x}^{1}(x, y)\right\rangle\right| \leqq C\left|a_{m}^{0}(0)\right| \lambda_{m}^{3} \sum_{s \neq m} \frac{\lambda_{s}^{2}}{\left|\lambda_{m}^{2}-\lambda_{s}^{2}\right|^{3}} \\
& \left|\left\langle\Phi_{m}(x, y), v_{m, x x}^{1}(x, y)\right\rangle\right| \leqq C\left|a_{m}^{0}(0)\right| \lambda_{m}^{3} \sum_{s \neq m} \frac{\lambda_{s}^{2}}{\left|\lambda_{m}^{2}-\lambda_{s}^{2}\right|^{3}}\left[1+\frac{\lambda_{m}^{2}+\lambda_{s}^{2}}{\left|\lambda_{m}^{2}-\lambda_{s}^{2}\right|}\right] \\
& \left|\left\langle\Phi_{m, x}(x, y), v_{m, x}^{1}(x, y)\right\rangle\right| \leqq C\left|a_{m}^{0}(0)\right| \lambda_{m}^{3} \sum_{s \neq m} \frac{\lambda_{s}^{2}}{\left|\lambda_{m}^{2}-\lambda_{s}^{2}\right|^{3}}\left[1+\frac{\lambda_{m}^{2}+\lambda_{s}^{2}}{\left|\lambda_{m}^{1}-\lambda_{s}^{2}\right|}\right]
\end{aligned}
$$

These estimates in turn lead directly to

$$
\begin{aligned}
& \left|a_{m}^{1}(0)\right| \leqq C \lambda_{m} \sum_{s \neq m} \frac{\lambda_{s}^{2}\left|a_{s}^{0}(0)\right|}{\left|\lambda_{m}^{2}-\lambda_{s}^{2}\right|^{2}} \\
& \left|A_{m}^{1}(x)\right| \leqq C \lambda_{m}\left|a_{m}^{0}(0)\right|\left(1+\lambda_{m}^{2} \sum_{s \neq m} \frac{\lambda_{s}^{2}}{\left|\lambda_{m}^{2}-\lambda_{s}^{2}\right|^{3}}\right) \\
& \left|\dot{A}_{m}^{1}(x)\right| \leqq C \lambda_{m}\left|a_{m}^{0}(0)\right|\left(1+\lambda_{m}^{2} \sum_{s \neq m} \frac{\lambda_{s}^{2}}{\left|\lambda_{m}^{2}-\lambda_{s}^{2}\right|^{3}}\left[1+\frac{\lambda_{m}^{2}+\lambda_{s}^{2}}{\left|\lambda_{m}^{2}-\lambda_{s}^{2}\right|}\right]\right)
\end{aligned}
$$

so that both $a_{m}^{1}$ and $\dot{a}_{m}^{1}$ satisfy

$$
\left\{\begin{array}{l}
\left|a_{m}^{1}(x)\right| \\
\left|\dot{a}_{m}^{1}(x)\right|
\end{array}\right\} \leqq C \lambda_{m}\left(\left|a_{m}^{0}(0)\right| 1+\lambda_{m}^{2} \sum_{s \neq m} \frac{\lambda_{s}^{2}}{\left|\lambda_{m}^{2}-\lambda_{s}^{2}\right|^{3}}+\sum_{s \neq m} \frac{\lambda_{s}^{2}\left|a_{s}^{0}(0)\right|}{\left|\lambda_{m}^{2}-\lambda_{s}^{2}\right|^{2}}\right)
$$

and

$$
\left|\ddot{a}_{m}^{1}(x)\right| \leqq C\left\{\left|a_{m}^{1}(x)\right|+\left|a_{m}^{0}(0)\right| \lambda_{m}\left(1+\lambda_{m}^{2} \sum_{s \neq m} \frac{\lambda_{s}^{2}}{\left|\lambda_{m}^{2}-\lambda_{m}^{2}\right|^{3}}\left[1+\frac{\lambda_{m}^{2}+\lambda_{s}^{2}}{\left|\lambda_{m}^{2}-\lambda_{s}^{2}\right|}\right]\right)\right\}
$$

We come now to the key estimate for the series defining $\tilde{V}^{1}$. Let $D^{2} V$ denote any derivative up through the second order of term-wise differentiation of $\tilde{V}^{1}$. 
Then we have after a lengthy computation

$$
\begin{aligned}
\left|D^{2} V\right| \leqq & C \sum_{m=1}^{\infty} \lambda_{m}^{3} \sum_{s \neq m} \frac{\lambda_{s}^{2}\left|a_{s}^{0}(0)\right|}{\left|\lambda_{m}^{2}-\lambda_{s}^{2}\right|} \\
& +C \sum_{m=1}^{\infty} \lambda_{m}^{2}\left|a_{m}^{0}(0)\right|\left\{\lambda_{m}+\sum_{s \neq m} \frac{\lambda_{s}\left(\lambda_{m}^{2}+\lambda_{s}^{2}\right)}{\left|\lambda_{m}^{2}-\lambda_{s}^{2}\right|^{2}}\left[1+\frac{\lambda_{m}^{2}}{\left|\lambda_{m}^{2}-\lambda_{s}^{2}\right|}+\frac{\lambda_{m} \lambda_{s}}{\left|\lambda_{m}^{2}-\lambda_{s}^{2}\right|^{2}}\right]\right\}
\end{aligned}
$$

uniformly on $\bar{\Omega}$. The constant $C$ may depend upon $\varepsilon$.

Acknowledgements. I want to express my appreciation to Professor Wolfgang Wendland and his colleagues at Darmstadt for their kind encouragement and hospitality during my stay there. I wish to thank Professor Manfred Schneider of the University of Karlsruhe for valuable suggestions in initial stages of this work. The perceptive remarks of Dr. A. D. Gilbert of the University of Edinburgh helped me to detect an error in the original version of the manuscript. He was also instrumental in improving the presentation of the results by his thoughtful suggestions.

\section{REFERENCES}

1. A. V. Bitsadze, Equations of the Mixed Type (Pergamon Press, New York, 1964).

2. W. EcKhaus and E. M. DE JAGER, Asymptotic solutions of singular perturbation problems for linear differential equations of elliptic type, Arch. Rational Mech. and Anal. 23 (1966), 26-86.

3. E. M. DE J J 12 (1975), 145-171.

4. G. C. Hsiao and R. J. Weinacht, Singular perturbation problems for a class of singular partial differential equations, Lecture Notes in Mathematics 564 (1976), 249-258.

5. G. E. LATTA, Singular Perturbation Problems (Ph.D. Thesis, California Institute of Technology, 1951).

6. J. B. Keller, Perturbation Theory (Lecture Notes, Mathematics Department, Michigan State University, 1968).

7. E. Hopf, A remark on linear elliptic equations of second order, Proc. Amer. Math. Soc. 3 (1952), 791-793.

University of Delaware

Ni:wark, Delaware 19711, USA

AND

Fachbereich Mathematik

Technische Hochschule Darmstadt

61 Darmstadt, West Germany 